\title{
Effect of phospholipid composition on the structure and physicochemical stability of proliposomes incorporating curcumin and cholecalciferol
}

Chaves, M.A. ${ }^{a}$; Pinho, S.C. ${ }^{\mathrm{a}^{*}}$

a Department of Food Engineering, School of Animal Science and Food Engineering, University of São Paulo, Av Duque de Caxias Norte 225, Pirassununga, SP, Brazil

*E-mail of the corresponding author: samantha@usp.br

\begin{abstract}
Proliposomes are dry phospholipid-based particles in which bioactives can be entrapped, and that can produce liposomal suspensions if adequately hydrated. In our study, curcumin and cholecalciferol were incorporated in proliposomes obtained by coating of micronized sucrose. Different mass ratios of Lipoid $\mathrm{S} 40$ and Phospholipon $90 \mathrm{H}$ were used to produce the proliposomes. The powders were structurally characterized and bioactives content were analyzed over 60 days of storage. Curcumin and cholecalciferol amounts in F100CV formulation were 100 and $98.7 \%$ of their initial amount, respectively. Strucutral characterization showed bioactives were successfully incorporated in concentrations compatible with recommended daily dosages.
\end{abstract}

Keywords: proliposomes, curcuminoid, vitamin $D_{3}$, Raman spectroscopy, powder characterization 


\section{Introduction}

Proliposomes are defined as dry, free-flowing powders composed mainly of phospholipids and that can instantly form liposome dispersions upon addition of an aqueous solution under appropriate conditions [1]. Advantages attributed to proliposomes as the convenience for transportation, distribution, and dosing, including the production of capsules/tablets, easier to be industrialized [2].

Curcumin is the major yellow hydrophobic pigment of the rhizomes of Curcuma longa. Nowadays, despite its use as food spicy, curcumin has been studied by pharmaceutical and medical researchers because of its antioxidant, anti-inflammatory and anti-carcinogenic actions [3]. However, the main limitation for the application of curcumin in food is its low water solubility and its poor bioavailability [4]. Cholecalciferol (vitamin $\mathrm{D}_{3}$ ) is the naturally occurring form of vitamin D. It plays an important role in regulation of calcium homeostasis and mineralization of bones [5]. Unfortunately, vitamin $\mathrm{D}_{3}$ carency affects almost $50 \%$ of worldwide population due, mostly, to lifestyle habits and environment factors that reduce the exposition to sunlight [6]. Thus, its supplementation is highly necessary as few enriched food are commercially available. However, as curcumin, vitamin $D_{3}$ is extremely sensitive to higher temperatures and exposure to light [7]. Hence, encapsulation in lipid delivery system could increase their stability and bioacessibility $[8,9]$.

In this study, curcumin and cholecalciferol were incorporated in proliposomes obtained by coating of micronized sucrose. The powders were characterized by Raman spectroscopy (RS) and Fourier-transform infrared spectroscopy (FTIR). In addition, proliposomes were analyzed in terms of their morphology, hygroscopicity, solubility, and amount of bioactives retained. Data revealed by this research will help to show the feasibility to produce proliposomes containing both bioactives in concentrations compatible with their recommended daily dosage for further application in food formulations.

\section{Materials and Methods}

\subsection{Production of proliposomes by coating of micronized sucrose}

Proliposomes production was based in the methodology described by Silva et al. (2017) [10]. Different mass percentages of phospholipids were tested: 50\% Lipoid S40 + 50\% Phospholipon 90H (F50), 70\% Lipoid S40 + 30\% Phospholipon 90H (F70), and 100\% Lipoid S40 (F100).

\subsection{Spectroscopical characterizaton of the proliposomes}

The spectra in the infrared regions were obtained in the 4000-400 $\mathrm{cm}^{-1}$ region by using a Perkin Elmer FT-IR Spectrometer (Waltham, MA, USA). Raman spectroscopy analyses 
were performed with a RAMII (Billerica, MA, USA) in the 4000-400 $\mathrm{cm}^{-1}$ region with Ge detector and a $1064 \mathrm{~nm}$ laser.

\subsection{Morphololgy of proliposomes by scanning electron microscopy (SEM)}

Morphology was studied using a scanning electron microscope (model 3000 TM; Hitachi, Japan) equipped with TM3000 software.

\subsection{Hygroscopicity and solubility}

The proliposomes were characterised in terms of hygroscopicity according to the procedure described by Cai and Corke (2000) [11]. The solubility of the proliposomes was determined according to the method of Eastman \& Moore (1984) [12].

\subsection{Quantification of bioactives in proliposomes}

Curcumin quantification was carried out according to Silva et al. (2017) [10]. Concentrations were obtained by absorbance readings at $425 \mathrm{~nm}$ in a spectrophotometer (Libra S22; Biochrom, Cambridge, UK). Regarding cholecalciferol retained in proliposomes, these values were obtained by high-performance liquid chromatography (Shmadzu; Kyoto, Japan) according to Staffas and Nyman (2003) [13].

\section{Results and Discussion}

\subsection{Visual aspect and morphology of proliposomes}

The visual aspect of blank and curcumin-cholecalciferol-loaded proliposomes are shown in Figure 1. The powders containing curcumin presented an intense and characteristic yellow color. The formulation prepared with only Lipoid S40 (F100) seemed to be more agglomerated than the produced with Phospholipon $90 \mathrm{H}$. This may be due because Lipoid S40 is a non-hydrogenated phospholipid, with a strong tendency to absorve moisture from the environment.

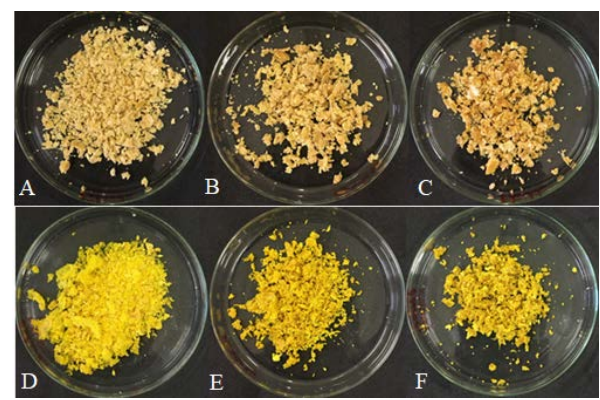

Figure 1. Visual aspects of blank (superior line) and curcumin-cholecalciferol-entrapped (inferior line) proliposomes produced with (A, D) 50\% Lipoid S40; (B, E) 70\% Lipoid S40; (C, F) 100\% 
The coating of micronized sucrose by phospholipids produced flake powders whose morphological characteristics are shown in the scanning electron micrographies illustrated in Fig. 2. In the SEM micrographs, it is possible to distinguish a layer of phospholipid coating the micronized sucrose granules. The coated material presented very irregular and different shapes, with many agglomerates in the visual fields.
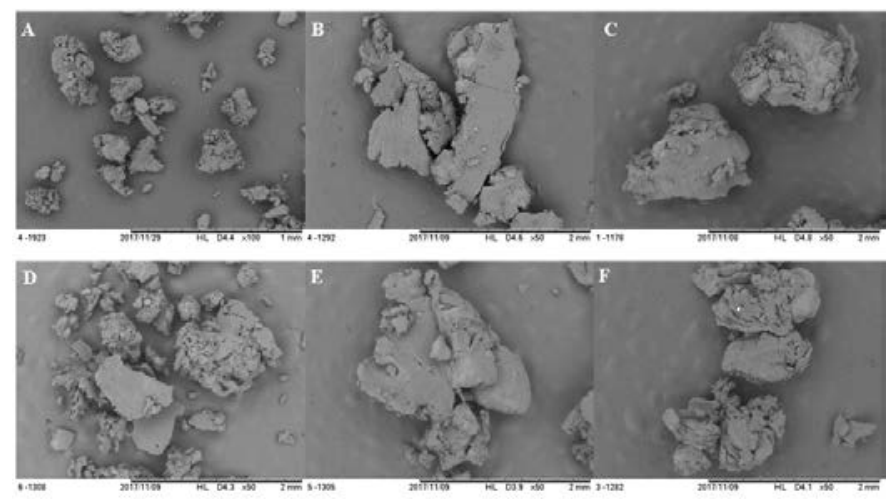

Figure 2. Micrographies obtained by scanning electronic spectroscopy for the blank (superior line) and curcumin-cholecalciferol-entrapped (inferior line) proliposomes produced with $(A, D) 50 \%$

Lipoid S40; (B, E) 70\% Lipoid S40; (C, F) 100\% Lipoid S40. Scale: 2nm. Magnitude: 50x

\subsection{Fourier-transform infrared spectroscopy (FTIR)}

The characteristic absorption peaks for blank and loaded proliposomes obtained by FTIR are shown in Figure 3. No major changes were detected in the spectra of empty and loaded proliposomes. Intense vibration peaks were observed at 2854 and $2920 \mathrm{~cm}^{-1}$ in all spectra. These peaks are related to ${ }_{s} v\left(\mathrm{CH}_{2}\right)$, and the higher their intensity, the more ordered are the acyl chains of the phospholipids. Therefore, as shown in Figs. 3E and 3F, higher amounts of Lipoid S40 in the proliposomes led to a reduction in the peak intensities. Such reduction was mainly due to the high content of unsaturated phospholipids in Lipoid S40, which helps to decrease the acyl chains ordering [10]. On the other hand, assymetrical stretching mode of a $\mathrm{P}=\mathrm{O}$ group is identified by a peak in the range $1200-160 \mathrm{~cm}^{-1}$ according to Lewis and McElhaney (1996) [14]. In this case, proliposomes showed this peak as the located in the wavenumber near $1043 \mathrm{~cm}^{-1}$. Popova and Hincha (2003) [15] stated this peak increased its position with hydrogen bonding. Regarding these peaks in Figs $3 \mathrm{E}$ and $3 \mathrm{~F}$, it can be verified a slight decrease, an indication that part of sucrose interacted with the polar heads of phospholipids, in a behavior that resembles that in which sucrose is used as cryoprotector during the production of lyophilized liposomes [16]. These interactions caused also a decrease in $\mathrm{C}=\mathrm{O}$ stretching, which was located at $1741 \mathrm{~cm}^{-1}$. Summarily, it can be stated that part of sucrose interacted with some polar heads of the phospholipids presented in Lipoid S40, hindering the coating process [10]. 


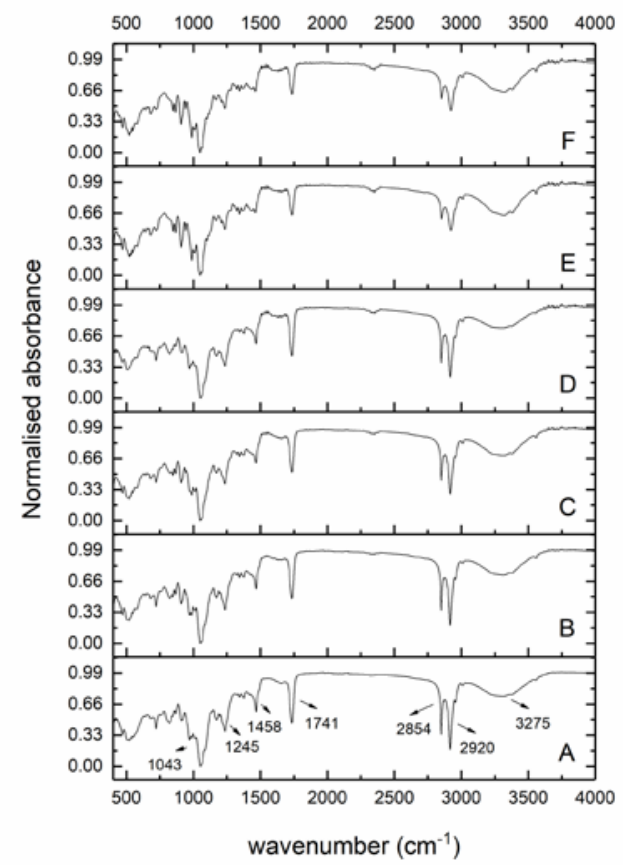

Figure 3. FTIR spectra obtained for formulations F50 (A); F50CV (B); F70 (C); F70CV (D); F100 (E) and F100CV (F).

\subsection{Raman spectroscopy}

Figure 4 shows the FT-Raman spectra obtained for blank and loaded proliposomes. The bands at 1596 and $1632 \mathrm{~cm}^{-1}$, are attributed to $\mathrm{v}(\mathrm{C}=\mathrm{C})$ motions of the inter-ring chain of curcumin and to $\mathrm{v}(\mathrm{C}=\mathrm{O}) / \mathrm{v}(\mathrm{C}=\mathrm{C})$ vibrations in the side aromatic rings of both curcumin and cholecalciferol. As can be seen, these peaks appeared only on formulations F50CV and F70CV. Therefore, it can be stated that in formulation F100CV both bioactives remained entrapped in the phospholipid matrix in their amorphous form. Changes in the broadening of the 1596 and $1632 \mathrm{~cm}^{-1}$ bands seen between F50CV and F70CV are due to conformational modifications on the side aromatic rings that can cause a variation of the electronic conjugation degree along the entire molecule [17]. Other Raman peak assignments for curcumin were obtained by Mohan et al. (2012) [18] and appeared in F50CV and F70CV spectras, as it follows: $1125 \mathrm{~cm}^{-1}(\delta(\mathrm{CCH})$ of aromatic rings and $\delta(\mathrm{C}-$ $\mathrm{OH})$ of the enolic group coupled to $\delta(\mathrm{C}=\mathrm{CH})$ in the inter-ring chain $), 1241 \mathrm{~cm}^{-1}(\delta(\mathrm{CH})$ of the aromatic rings, combined to $v(\mathrm{C}-\mathrm{O})$ of the ether groups linked to these rings), $1295 \mathrm{~cm}^{-}$ ${ }^{1}\left(\delta(\mathrm{CCH})\right.$ of the inter-ring chain $\left(\mathrm{C}^{10}\right.$ and $\left.\mathrm{C}^{11}\right)$, and $1439 \mathrm{~cm}^{-1}(\delta(\mathrm{CCC}), \delta(\mathrm{CCH})$ and $\delta(\mathrm{C}-$ $\mathrm{OH})$ of aromatic rings). Cholecalciferol peak at $1650 \mathrm{~cm}^{-1}$ was possibly covered by peak at $1655 \mathrm{~cm}^{-1}$ commonly found in polyunsaturated lipids [19]. In addition, the low 
concentration of cholecalciferol added in proliposomes may have remained within the surrounding matrix, which explained the absence of its related peak.

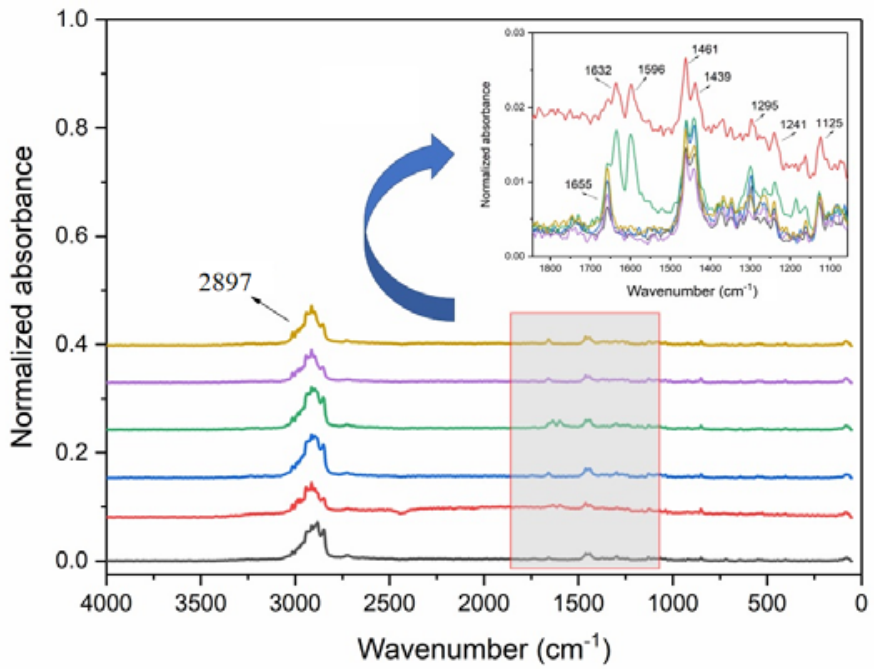

Figure 4. FT-Raman spectra obtained for formulations F50 (black); F50CV (red); F70 (blue); F70CV (green); F100 (purple) and F100CV (orange).

\subsection{Hygroscopicity, solubility and amount of bioactives retained}

Physicochemical parameters obtained for proliposomes over 60 days of storage are in Table 1. Higher hygroscopicities were obtained for proliposomes with higher contents of Lipoid S40. These values can be considered low, which is a highly desirable characteristic for powders. The incorporation of the hydrophobic actives in proliposomes did not lead to a decrease in solubility values. This result corroborates what was mentioned before, that both molecules remained entrapped in phospholipid matrix. However, the values obtained were quite lower than those obtatined by Silva et al. (2017) [10] which also produced proliposomes containing curcumin by coating of micronized method. Additionally, it is important to mention that Silva et al. (2017) [10] produced proliposomes using only Phospholipon $90 \mathrm{H}$, which contains a higher number of hydrogenated phospholipids, which are easier to be dispersed in aqueous medium.

The amount of bioactives retained in proliposomes after 60 days of storage are presented in Table 1 . Considering that curcumin and cholecalciferol are highly sensitive to degradation, the results obtained can be considered promising, as $100 \%$ of curcumin and more than $85 \%$ of the cholecalciferol initially present in the powder were preserved. 
Table 1. Physicochemical parameters obtained for blank and loaded proliposomes

\begin{tabular}{ccccc}
\hline & $\begin{array}{c}\text { Solubility } \\
(\%)\end{array}$ & $\begin{array}{c}\text { Hygroscopicity } \\
(\%)\end{array}$ & \multicolumn{2}{c}{$\begin{array}{c}\text { Preserved bioactives } \\
\text { (after 60 days) (\%) }\end{array}$} \\
\hline & & & Curcumin & Cholecalciferol \\
\hline F50 & $36.4^{\mathrm{C}} \pm 2.02$ & $7.24^{\mathrm{B}} \pm 0.23$ & - & - \\
\hline F50CV & $42.4^{\mathrm{B}} \pm 0.64$ & $5.90^{\mathrm{C}} \pm 0.23$ & $100 \pm 1.18$ & $92.3 \pm 3.50$ \\
\hline F70 & $44.5^{\mathrm{A}} \pm 0.93$ & $6.49^{\mathrm{C}} \pm 0.31$ & - & - \\
\hline F70CV & $44.8^{\mathrm{A}} \pm 1.38$ & $7.50^{\mathrm{BC}} \pm 1.47$ & $100 \pm 0.91$ & $85.1 \pm 0.10$ \\
\hline F100 & $37.9^{\mathrm{C}} \pm 1.36$ & $11.1^{\mathrm{A}} \pm 2.66$ & - & - \\
\hline F100CV & $42.5^{\mathrm{AB}} \pm 1.49$ & $8.62^{\mathrm{AB}} \pm 1.54$ & $100 \pm 1.04$ & $98.7 \pm 0.37$ \\
\hline
\end{tabular}

\section{Conclusions}

The experimental data indicate it is possible to produce proliposomes containing curcumin and cholecalciferol by coating of micronized sucrose. The process of coating with phospholipid was effective as illustrated by SEM images. Proliposomes presented low hygroscopicity and moderate solubility, which not hinders the production of liposome dispersions, as mechanical energy can be applied during their preparation. The amount of cholecalciferol added to the formulations was low, which hindered its determination by spectroscopies. The results showed the feasibility of producing proliposomes incorporating both bioactives in concentrations compatible with their recommended daily dosage for further application in food formulations.

\section{References}

[1] Payne, N.I.; Browning, I.; Hynes, C.A. Characterization of Proliposomes. Journal of Pharmaceutical Sciences 1986, 75 (4), 330-333.

[2] Patel, G.M.; Shelat, P.K.; Lalwani, A.N. QbD based development of proliposome of lopinavir for improved oral bioavailability. European Journal of Pharmaceutical Sciences 2017, 108, 50-61.

[3] Cuomo, F.; Cofelice, M.; Venditti, F.; Ceglie, A.; Miguel, M.; Lindman, B.; Lopez, F. In-vitro digestion of curcumin loaded chitosan-coated liposomes. Colloids and Surfaces B: Biointerfaces 2017. In Press.

[4] Zou, L.; Zheng, B.; Liu, W.; Liu, C.; Xiao, H.; McClements, D.J. Enhancing nutraceutical bioavailability using excipient emulsions: Influence of lipid droplet size on solubility and bioaccessibility of powdered curcumin. Journal of Functional Foods 2015, 15, 72-83.

[5] Picciano, M.F.; Dwyer, J.T.; Radimer, K.L. Dietary supplement use among infants, children and adolescents in the United States, 1999-2002. Archives of Pediatrics and Adolescent Medicine 2007, 161 (10), 978-985. 
[6] Holick, M.F. Vitamin D deficiency. The New England Journal of Medicine 2007, 357 (3), 266-281.

[7] Luo, Y.; Teng, Z.; Wang, Q. Development of zein nanoparticles coated with carboxymethyl chitosan for encapsulation and controlled release of vitamin D3. Journal of Agricultural and Food Chemistry 2012, 60 (3), 836-843.

[8] Park, S.J.; Garcia, C.V.; Shin, G.H.; Kim, J.T. Development of nanostructured lipid carriers for the encapsulation and controlled release of vitamin D3. Food Chemistry 2017, 225, 213-219.

[9] Jin, H-H.; Lu, Q.; Jiang, J-G. Curcumin liposomes prepared with milk fat globule membrane phospholipids and soybean lecithin. Journal of Dairy Science 2016, 99 (3), 1780-1790.

[10] Silva, G.G.; Jange, C.G.; Rocha, J.S.S.; Chaves, M.A.; Pinho, S.C. Characterisation of curcumin-loaded proliposomes produced by coating of micronised sucrose and hydration of phospholipid powders to obtain multilamellar liposomes. International Journal of Food Science and Technology 2017, 52 (3), 772-780.

[11] Cai, Y.Z.; Corke, H. Production and properties of spray-dried Amaranthus betacyanin pigments. Journal of Food Science 2000, 65 (7), 1248-1252.

[12] Eastman, J.E.; Moore, C.O. Cold Water Soluble Granular Starch for Gelled Food Composition. U.S. Patent 4465702, 14 ago. 1984.

[13] Staffas, N.; Nyman, A. Determination of cholecalciferol (vitamin D3) in selected foods by liquid chromatography: NMKL collaborative study. Journal of AOAC International 2003, 86 (2), 400-406.

[14] Lewis, R.N.; McElhaney, R.N. Fourier transform infrared spectroscopy in the study of hydrated lipids and lipid bilayer membranes. In Infrared Spectroscopy of Biomolecules (Mantsch, H.H. \& Chapman, D., editors), Wiley, Chichester, 159-202.

[15] Popova, A.V.; Hincha, D.K. Intermolecular Interactions in Dry and Rehydrated Pure and Mixed Bilayers of Phosphatidylcholine and Digalactosyldiacylglycerol: A Fourier Transform Infrared Spectroscopy Study. Biophysical Journal 2003, 85 (3), 1682-1690.

[16] Doxastakis, M.; Sum, A.K.; Pablo, J.J. Modulating Membrane Properties: The Effect of Trehalose and Cholesterol on a Phospholipid Bilayer 2005, 109 (50), 24173-24181.

[17] Castillo, M.L.R.; López-Tobar, E.; Sanchez-Cortes, S.; Flores, G.; Blanch, G.P. Stabilization of curcumin against photodegradation by encapsulation in gammacyclodextrin: A study based on chromatographic and spectroscopic (Raman and UVvisible) data. Vibrational Spectroscopy 2015, 81, 106-111.

[18] Mohan, P.R.K.; Sreelakshmi, G.; Muraleedharan, C.V.; Joseph, R. Water soluble complexes of curcumin with cyclodextrins: Characterization by FT-Raman spectroscopy. Vibrational Spectroscopy 2012, 62, 77-84.

[19] Schultz, Z.D. Raman Spectroscopy and Imaging of Biomolecules using Targeted Nanoparticles. Biophysical Journal 2012, 102 (3), 201a. 